\title{
Study of the di-lepton final state with missing transverse momentum with the ATLAS detector
}

\author{
Artem Basalaev*, on behalf of the ATLAS Collaboration \\ National Research Centre "Kurchatov Institute” B.P.Konstantinov Petersburg Nuclear Physics \\ Institute, St. Petersburg, Russia \\ E-mail: artem.basalaev@cern.ch
}

\begin{abstract}
Common analysis techniques are reviewed for di-lepton searches with large missing transverse momentum in proton-proton collisions in ATLAS detector at the LHC. Background estimates and corresponding systematic uncertainties are shown. The presented analyses are the search for an additional heavy Higgs boson with a mass in the range between 300 and $1000 \mathrm{GeV}$ decaying into $Z$ boson pairs with two leptons and two neutrinos in the final state, the search for invisibleparticle decay modes of a Higgs boson with a mass of $125 \mathrm{GeV}$ produced in association with a $Z$ boson, and the search for dark matter particle candidates produced in association with a $Z$ boson. Results are presented for $8 \mathrm{TeV}$ proton-proton collisions, except for the search for heavy Higgs bosons, for which $13 \mathrm{TeV}$ results are shown.
\end{abstract}

Fourth Annual Large Hadron Collider Physics

13-18 June 2016

Lund, Sweden

${ }^{*}$ Speaker. 


\section{Introduction}

In proton-proton collisions physics processes that give rise to a final state with two leptons $\left(e^{+} e^{-}\right.$or $\left.\mu^{+} \mu^{-}\right)$plus a missing transverse momentum $\left(E_{\mathrm{T}}^{\mathrm{miss}}\right)$ are very common. These include the recently discovered Higgs boson and many other predicted processes that fall into the field of Beyond Standard Model (BSM) physics or Dark Matter sector (DM).

One of these promising searches is a search for new high-mass resonances decaying into two $Z$ bosons in the decay mode $H \rightarrow Z Z \rightarrow \ell^{+} \ell^{-} v \bar{v}$ [1]. Some extensions to the SM that have an extended scalar sector predict additional heavy Higgs bosons. Two models were considered that predict an additional Higgs boson are the two-Higgs-doublet model (2HDM) [2] and the electroweaksinglet (EWS) model [3]. In addition, the observed missing transverse $\operatorname{mass}^{1}\left(m_{\mathrm{T}}\right)$ distribution was used to set upper limits on the production cross-section of the graviton candidate $\left(G^{*}\right)$ predicted by the bulk Randall-Sundrum (RS) model [4].

At hadron colliders, weakly interacting massive particles (WIMPs), the promising candidates for dark matter particles, are predicted to be produced in pairs in some models. A WIMP would escape as undetected particle, but could be identified by a signature involving missing transverse momentum $E_{\mathrm{T}}^{\text {miss }}$. Selecting events with a clear $Z$ boson signature results in well-studied $E_{\mathrm{T}}^{\text {miss }}$ distribution. A search was performed for deviations from SM $E_{\mathrm{T}}^{\text {miss }}$ distribution predictions caused by extra contributions which can be calculated by specific effective field theory [7] and mediator models [8] for WIMP DM production associated with a $Z$ boson (Mono- $Z$ signature) [5].

The SM Higgs boson has a very small branching ratio (BR) to invisible decay, e.g. $B R(H \rightarrow$ $Z Z \rightarrow 4 v) \approx 0.001$. However, BSM theories predict the decay of the Higgs boson to stable or longlived particles that can not be detected by the ATLAS detector [6], which leads to the enhancement of the Higgs invisible decay BR (e.g. [9]). Search for invisible-particle decay modes of a Higgs boson with a mass of $125 \mathrm{GeV}$ produced in association with a $\mathrm{Z}$ boson in the $\mathrm{ZH}, \mathrm{H} \rightarrow$ invisible channel was performed [10]. In addition limits were placed on the cross section times branching ratio of any additional Higgs bosons decaying predominantly to invisible particles.

\section{Event Selection and Background Estimation}

Electron candidates are reconstructed from isolated energy deposits in the electromagnetic calorimeter with a shower shape consistent with electrons or photons, matched to inner detector tracks [11]. Muon candidates are reconstructed combining tracks independently found in the muon spectrometer and inner tracking detector [12]. Jets are reconstructed using the anti- $k_{t}$ algorithm [13] with a radius parameter $\mathrm{R}=0.4$. Basic kinematic cuts are also applied, along with some additional selection to ensure good separation between reconstructed objects $[1,5,10]$. The invariant mass of a lepton pair is required to be consistent with that of a $Z$ boson, $76 \mathrm{GeV} \leq \mathrm{M}_{\ell \ell} \leq 106 \mathrm{GeV}$. Events are required to pass event quality checks for errors in sub-detectors during recording, single-lepton trigger requirements, or lepton-pair trigger requirements (analysis-specific), and specific selection cuts in order to minimize the backgrounds, which are summarized in Table 1.

$$
{ }^{1} \text { Defined here as }\left(m_{\mathrm{T}}^{Z Z}\right)^{2} \equiv\left(\sqrt{m_{Z}^{2}+\left|\vec{p}_{\mathrm{T}}^{\ell \ell}\right|^{2}}+\sqrt{m_{Z}^{2}+\left|\vec{E}_{\mathrm{T}}^{\mathrm{miss}}\right|^{2}}\right)^{2}-\left|\vec{p}_{\mathrm{T}}^{\ell \ell}+\vec{E}_{\mathrm{T}}^{\text {miss }}\right|^{2}[1] \text {. }
$$


A lower threshold is set on the $E_{T}^{\text {miss }}$ variable, which helps rejecting the inclusive $Z$ production. To account for boosted $Z$ boson, analysis-specific cuts on lepton pair are introduced to ensure that leptons are close in space. The $E_{T}^{m i s s}$ is expected to be back-to-back with the $Z$ boson. This is accounted for in the $\Delta \phi\left(Z, E_{T}^{m i s s}\right)$ variable, on which a lower threshold is set. Any possible imbalance, implying the presence of fake $E_{\mathrm{T}}^{\text {miss }}$, is accounted for in the fractional $p_{\mathrm{T}}$ difference variable, defined as $\left|E_{T}^{\text {miss }}-p_{T}^{\ell \ell}\right| / p_{T}^{\ell \ell}$; for the search for additional heavy $H$, the transverse momentum of the jets is added to $E_{T}^{\text {miss }}$ for fractional $p_{\mathrm{T}}$ difference calculation. Additionaly for Mono- $Z$ search a cut on the pseudorapidity of the di-lepton system $\left|\eta_{l l}\right|$ is applied. For $Z H, H \rightarrow$ invisible search, possible misreconstructed energy in the calorimeter is also accounted for by using track-based $E_{\mathrm{T}}^{\text {miss }}$ and setting an upper threshold on $\Delta \phi\left(E_{T}^{\text {miss }}\right.$, Track $\left.E_{T}^{\text {miss }}\right)$. In order to suppress the DrellYan background contamination in the high $m_{T}$ region for the heavy $H$ search, two additional cuts, $\Delta \phi\left(\right.$ jets, $\left.E_{\mathrm{T}}^{\mathrm{miss}}\right)$ and $p_{T}^{Z} / m_{T}$, are implemented. Events are removed if they contain b-tagged jets (in case of search for heavy $H$ ) or jets with $p_{\mathrm{T}}>25 \mathrm{GeV}$ (in case of $Z H, H \rightarrow$ invisible and Mono- $Z$ searches) to suppress top-quark pair background. Events containing a third lepton with $p_{\mathrm{T}}>7$ $\mathrm{GeV}$, satisfying looser identification requirements are removed to suppress diboson background. Analysis-specific selections are motivated by optimization studies done using Monte Carlo (MC) simulation to ensure maximum signal yields while minimizing backgrounds.

\begin{tabular}{|l|l|l|}
\hline Heavy $H$ & $Z H, H \rightarrow$ inv. & Mono- $Z$ \\
\hline$E_{T}^{\text {miss }}>120 \mathrm{GeV}$ & $E_{T}^{\text {miss }}>90 \mathrm{GeV}$ & $E_{T}^{\text {miss }}>150,250,350,450 \mathrm{GeV}$ \\
$\left|\Delta R_{l l}\right|<1.8$ & $\left|\Delta \phi_{l l}\right|<1.7$ & $\left|\eta_{l l}\right|<2.5$ \\
$\Delta \phi\left(Z, E_{T}^{\text {miss }}\right)>2.7$ & $\Delta \phi\left(Z, E_{T}^{\text {miss }}\right)>2.6$ & $\Delta \phi\left(Z, E_{T}^{\text {miss }}\right)>2.5$ \\
$\mid E_{T}^{\text {miss }}$ with jet corr. $-p_{T}^{\ell \ell} \mid / p_{T}^{\ell \ell}<0.2$ & $\left|E_{T}^{\text {miss }}-p_{T}^{\ell \ell}\right| / p_{T}^{\ell \ell}<0.2$ & $\left|E_{T}^{\text {miss }}-p_{T}^{\ell \ell}\right| / p_{T}^{\ell \ell}<0.5$ \\
b-tagged jet veto & jet veto $p_{\mathrm{T}}>25 \mathrm{GeV}, \eta<2.5$ & jet veto $p_{\mathrm{T}}>25 \mathrm{GeV}, \eta<2.5$ \\
$\Delta \phi$ jets $\left.\left[p_{\mathrm{T}}>100 \mathrm{GeV}\right], E_{\mathrm{T}}^{\text {miss }}\right)>0.4$ & $\Delta \phi\left(E_{T}^{\text {miss }}\right.$, Track $\left.E_{T}^{\text {miss }}\right)<0.2$ & \\
$p_{T}^{Z} / m_{T}<0.7$ & & \\
\hline
\end{tabular}

Table 1: Analysis-specific event selection $[1,5,10]$.

The dominant background process is $Z Z \rightarrow \ell^{+} \ell^{-} v \bar{v}$, an irreducible background. Reducible backgrounds may have jets produced in association with two leptons, where the jets are misidentified or unreconstructed, such as $t \bar{t} \rightarrow \ell^{+} v b \ell^{-} \bar{v} \bar{b}, Z Z \rightarrow \ell^{+} \ell^{-} q \bar{q}, W Z \rightarrow \bar{q} q^{\prime} \ell^{+} \ell^{-}$or $Z+$ jets (including decays to $\tau$ leptons). The other important background is $W W \rightarrow \ell^{+} v \ell^{-} \bar{v}$, which may only be reduced through the mass window requirement. Additional reducible sources may produce events with more than two leptons where the additional leptons are misidentified or not reconstructed, or less than two leptons where jets are misidentified as leptons, such as $W Z \rightarrow \ell v \ell^{+} \ell^{-}$ and $W+$ jets, respectively.

Data-driven techniques for background estimation were used where possible. Otherwise, MC simulation samples were used, obtained with MC generators such as SHERPA, MADGrAPH, PowHEG. Detailed information on background estimations is provided in $[1,5,10]$.

The $Z Z$ and $W Z$ backgrounds are estimated from MC simulations. The $W W, t \bar{t}, W t$, and $Z \rightarrow t \bar{t}$ backgrounds are estimated from data using the absence of signal in the $e \mu$ channel and the relative production rate of $1: 1: 2$ for the ee, $\mu \mu$, and $e \mu$ channels. An $e \mu$ control region similar to the signal region is defined, and the background estimate for the ee and $\mu \mu$ signal regions is obtained 
from the number of $e \mu$ events in the control region after correcting for different acceptances and efficiencies. The $Z+$ jets background is estimated using data-driven techniques specific to the analysis (e.g. $A B C D$ method using uncorrelated variables [14]). The $W+$ jets background is estimated by reversing the electron isolation condition and loosening identification requirements for one electron in order to obtain a data sample enriched in jets reconstructed as electrons.

Theoretical and experimental systematic uncertainties are estimated for all studied backgrounds, including uncertainties associated with pileup reweighting, luminosity calculation, object and $E_{\mathrm{T}}^{\text {miss }}$ reconstruction algoritms and methods of background estimation. Detailed information on data yields, background estimates, associated statistical and systematic uncertainties can be found in $[1,5,10]$.

\section{Results and Conclusions}

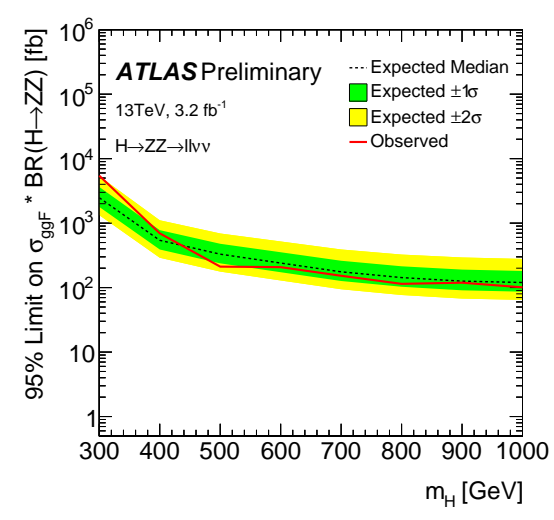

(a)

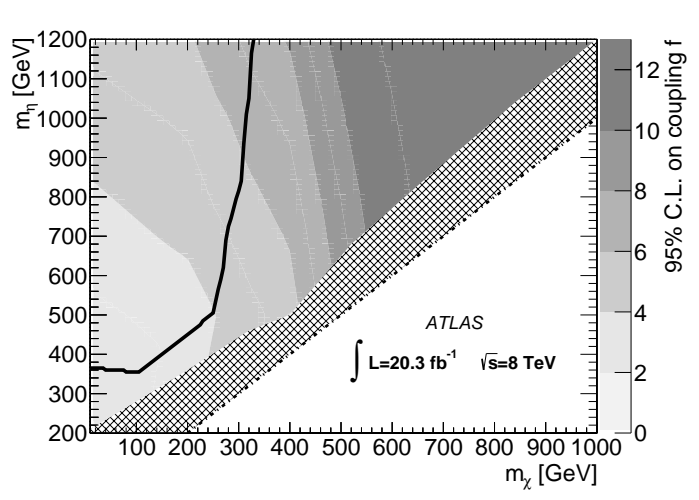

(c)

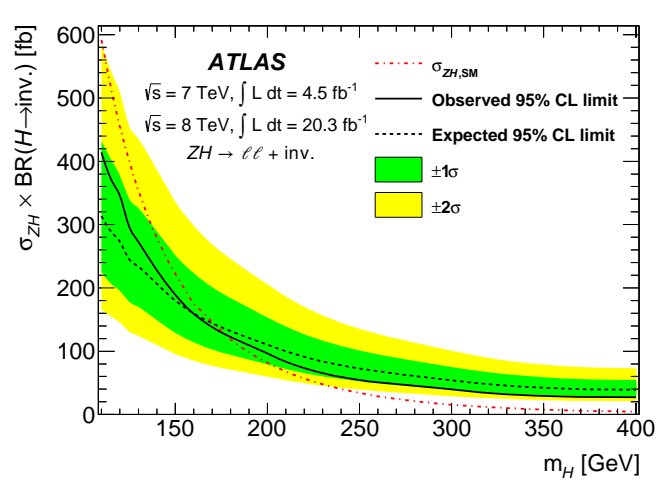

(b)

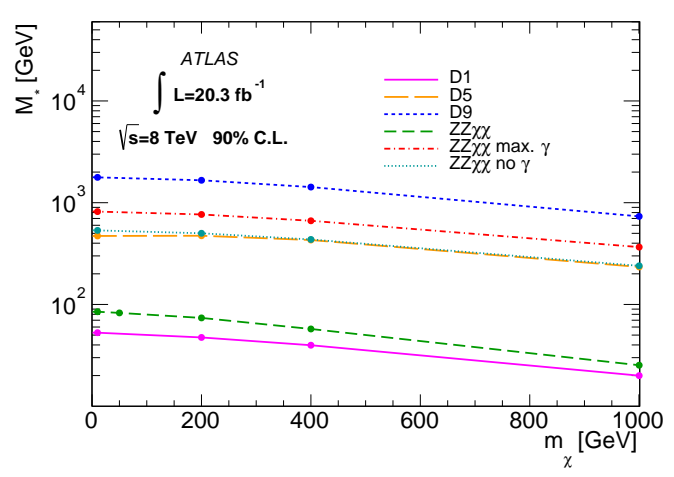

(d)

Figure 1: Observed upper limits at 95\% CL are shown: on the $\sigma(p p \rightarrow H) \times B R(H \rightarrow Z Z)$ for a narrowwidth, heavy-Higgs boson produced via gluon-gluon fusion [1] (a); on the $\sigma(p p \rightarrow Z H) \times B R(H \rightarrow$ inv) for a Higgs boson with $110<\mathrm{mH}<400 \mathrm{GeV}$ for the combined 7 and $8 \mathrm{TeV}$ data [10] (b); on the coupling constant $f$ of the scalar-mediator theory as a function of mediator mass $m_{\eta}$ and dark matter particle mass $m_{\chi}$ [5] (c). Observed $90 \%$ C.L. lower limits are shown on the mass scale $\mathrm{M}^{*}$ of considered effective field theories as a function of $m_{\chi}[5]$ (d). 
Search for additional heavy $H$ was performed on the data sample corresponding to an integrated luminosity of $3.2 \mathrm{fb}^{-1}$ collected by the ATLAS experiment at the LHC in 2015 in pp collisions at $\sqrt{s}=13 \mathrm{TeV}$. The limits on $\sigma(p p \rightarrow H) \times B R(H \rightarrow Z Z)$ at $95 \%$ confidence level (CL) are presented in Figure 1(a). ZH,H $\rightarrow$ invisible and Mono- $Z$ searches were performed on $20.3 \mathrm{fb}^{-1}$ of ATLAS dataset collected in 2012 at $\sqrt{s}=8 \mathrm{TeV}$. In addition, for $Z H, H \rightarrow$ invisible the $4.5 \mathrm{fb}^{-1}$ ATLAS dataset collected in 2011 at $\sqrt{s}=7 \mathrm{TeV}$ was used. The 95\% C.L. upper limits on $\sigma(p p \rightarrow Z H) \times B R(H \rightarrow i n v)$ are presented in Figure 1(b). The 95\% C.L. upper limits on the on the coupling constant $f$ of the scalar-mediator theory and the $90 \%$ C.L. lower limits on the mass scale $M^{*}$ of the effective field theory, which parameterizes the coupling between the WIMP and SM particles, are presented in Figure 1(c) and Figure 1(d) correspondingly. No significant deviations from the SM predictions have been observed in either of searches.

\section{References}

[1] ATLAS Collaboration, Search for high-mass resonances decaying into a $\mathrm{Z}$ boson pair in the $\ell \ell v \mathrm{v}$ final state in pp collisions at $\sqrt{s}=13 \mathrm{TeV}$ with the ATLAS detector, ATLAS-CONF-2016-012.

[2] G. Branco et al., Theory and phenomenology of two-Higgs-doublet models, Phys. Rept. 5161 (2012).

[3] A. Hill and J.J. van der Bij, Strongly interacting singlet-doublet Higgs model, Phys. Rev. D 363463 (1987).

[4] L. Randall and R. Sundrum, Large Mass Hierarchy from a Small Extra Dimension, Phys. Rev. Lett. 833370 (1999).

[5] ATLAS Collaboration, Search for dark matter in events with a $Z$ boson and missing transverse momentum in pp collisions at $\sqrt{s}=8 \mathrm{TeV}$ with the ATLAS detector,

Phys. Rev. D 90, 012004 (2014).

[6] ATLAS Collaboration, The ATLAS Experiment at the CERN Large Hadron Collider, JINST 3 S08003 (2008).

[7] J. Goodman, M. Ibe, A. Rajaraman, W. Shepherd, T. M. P. Tait, and H. B. Yu, Constraints on dark matter from colliders, Phys. Rev. D 82, 116010 (2010).

[8] N. Bell, J. Dent, A. Galea, T. Jacques, L. Krauss, and T. Weiler, Searching for dark matter at the LHC with a mono-Z, Phys. Rev. D 86, 096011 (2012).

[9] K. Griest and H. E. Haber, Invisible decays of Higgs bosons in supersymmetric models, Phys. Rev. D 37, 719 (1988).

[10] ATLAS Collaboration, Search for Invisible Decays of a Higgs Boson Produced in Association with a Z Boson in ATLAS, Phys. Rev. Lett. 112, 201802 (2014).

[11] ATLAS Collaboration, Electron performance measurements with the ATLAS detector using the 2010 LHC proton-proton collision data, Eur. Phys. J. C 72, 1909 (2012).

[12] ATLAS Collaboration, Measurement of the $W \rightarrow \ell v$ and $Z / \gamma * \rightarrow$ ll production cross sections in proton-proton collisions at $\sqrt{s}=7$ TeV with the ATLAS detector, J. High Energy Phys. 12060 (2010).

[13] M. Cacciari, G. P. Salam, and G. Soyez, NLO Higgs boson production via gluon fusion matched with shower in POWHEG, J. High Energy Phys. 04063 (2008).

[14] ATLAS Collaboration, A search for prompt lepton-jets in pp collisions at $\sqrt{s}=7 \mathrm{TeV}$ with the ATLAS detector, Phys. Lett. B 719, 299 (2013). 